\title{
Ultra-sensitive and compact on-chip gas sensor on a silicon nitride photonic integrated circuit
}

\author{
G. Antonacci ${ }^{1 *}$, J. Goyvaerts ${ }^{1 *}$, H. Zhao ${ }^{1}$, B. Baumgartner ${ }^{2}$, B. Lendl ${ }^{2}$, R. Baets ${ }^{1}$ \\ ${ }^{1}$ Photonics Research Group, Ghent University - Imec, Ghent, Belgium \\ ${ }^{2}$ Institute of Chemical Technologies and Analytics, TU Wien, Wien Austria
}

\section{Words Abstract}

We demonstrate a compact on-chip gas sensor based on a SiN photonic integrated circuit providing an estimated detection limit of $0.1 \mathrm{ppm}$ for a variety of gas vapors, including IPA, ethanol and acetone. The sensor consists of an integrated Mach-Zehnder interferometer having one arm coated with an absorptive mesoporous layer to enhance the interaction of the gas vapors with the waveguide evanescent field. Gas detection is accomplished by monitoring the spectral shift of the output signal induced by a change in the effective refractive index of the coated waveguide. Results pave the way to novel integrated solutions for environmental safety monitoring.

\section{Words Abstract}

An indispensable application where the interaction of light with its environment plays a substantial role is optical gas sensing. More particularly, compact and inexpensive gas sensors are of high interest for portable, cost effective, and real time sensing in a number of environmental and industrial applications. In this regard, optical structures fabricated on the Silicon Nitride technology provide a convenient platform for the implementation of highly compact, versatile and low-cost devices. Here, we demonstrate an ultra-sensitive on-chip gas sensor on a Silicon Nitride platform providing an unprecedented detection limit of $0.1 \mathrm{ppm}$. The sensor functionality relies on a change in the effective refractive index of the constituent SiN waveguides in response to the exposure to the gas vapors. In particular, the sensing arm of an integrated Mach-Zehnder interferometer is coated with a mesoporous silica layer that enables the absorption of the gas vapors in proximity of the waveguide. Light travelling along the sensing arm experiences a different refractive index when the gas is absorbed resulting in a wavelength shift at the output of the Mach-Zehnder interferometer. Detection of this spectral shift provides information about the presence of a gas vapor in the environment with concentrations as low as $0.1 \mathrm{ppm}$. We demonstrate this concept for a number of gas vapors such as IPA and ethanol. Results pave the way to disposable, compact and low cost environmental sensing applications. 
Google Deze pagina weergeven in het: Nedertands - Vertalen Uitschakelen voor Engels

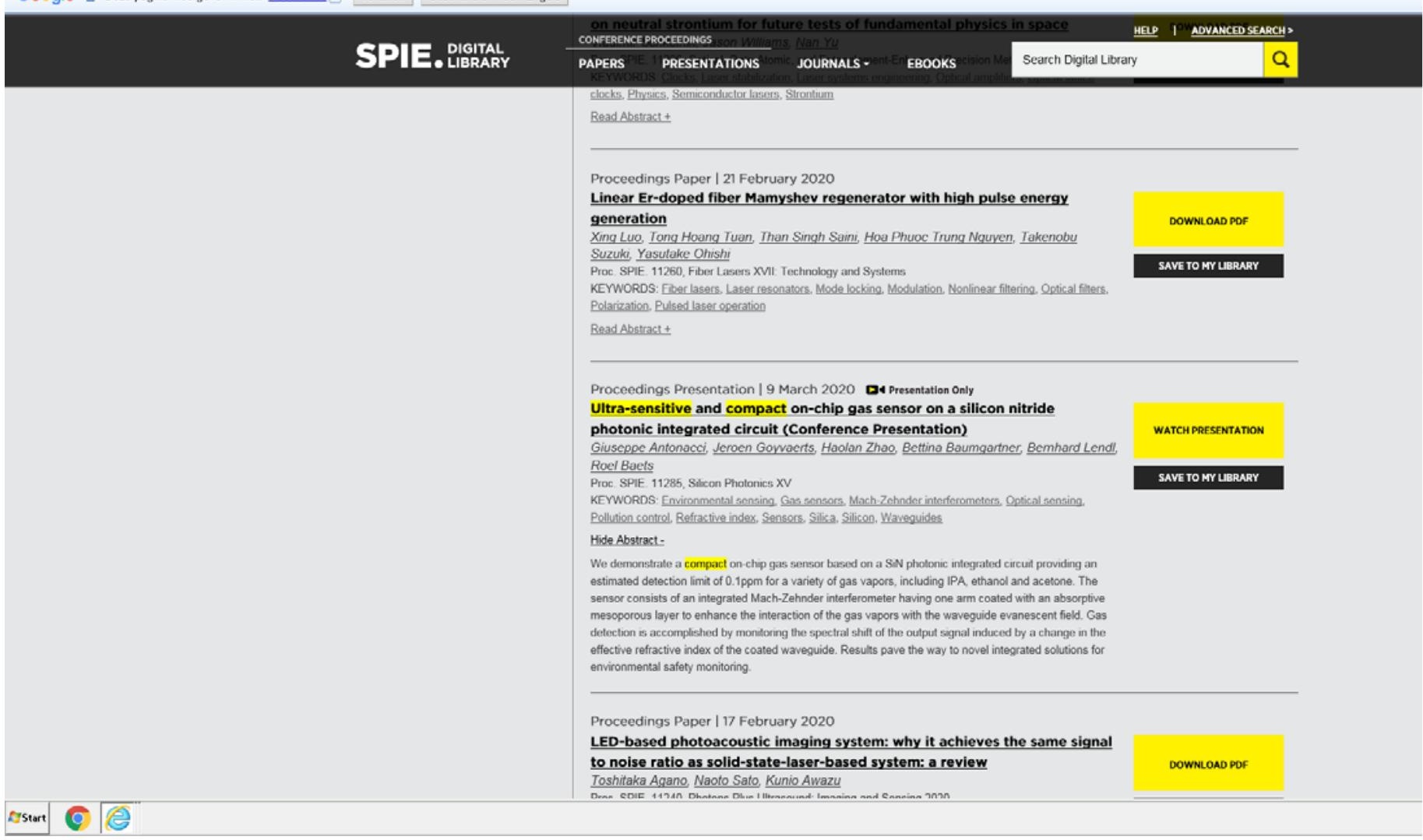

